\title{
Pterostilbeno Reduz o Estresse Oxidativo no Pulmão e no Ventrículo Direito Induzido por Infarto do Miocárdio Experimental
}

\author{
Pterostilbene Reduces Experimental Myocardial Infarction-Induced Oxidative Stress in Lung and Right Ventricle \\ Silvio Tasca, ${ }^{10}$ Cristina Campos, ${ }^{1}$ Denise Lacerda, ${ }^{1}$ Vanessa D. Ortiz, ${ }^{1}$ Patrick Turck, ${ }^{1}$ Sara E. Bianchi, ${ }^{2}$ Alexandre \\ L. de Castro, ${ }^{1}$ Adriane Belló-Klein, ${ }^{1}$ Valquiria Bassani, ${ }^{2}$ Alex Sander da Rosa Araújo ${ }^{1}$ \\ Programa de Pós-Graduação em Ciências Biológicas: Fisiologia, Instituto de Ciências Básicas da Saúde, Universidade Federal do Rio Grande \\ do Sul (UFRGS), 1 Porto Alegre, RS - Brasil \\ Programa de Pós-Graduação em Ciências Farmacêuticas, Universidade Federal do Rio Grande do Sul (UFRGS), 2 Porto Alegre, RS - Brasil
}

\section{Resumo}

Fundamento: O pterostilbeno (PS), um composto polifenólico natural e antioxidante, surge como uma intervenção promissora para minimizar danos do infarto agudo do miocárdio (IAM).

Objetivo: Este estudo teve como objetivo avaliar o desempenho do PS na promoção da homeostase redox nos pulmões e no ventrículo direito (VD) de animais infartados.

Métodos: Ratos Wistar machos (60 dias de idade) foram randomizados em três grupos: SHAM, IAM (infarto) e IAM+PS (IAM + pterostilbeno). Sete dias após o procedimento de IAM, os ratos foram tratados com PS (100 mg/kg/dia) por gavagem por oito dias. Os animais foram depois sacrificados e os pulmões e VD foram coletados para análise do balanço redox (diferenças foram consideradas significativas quando $p<0,05$ ).

Resultados: Nossos resultados mostram que o IAM desencadeia a interrupção redox no VD e nos pulmões, o que pode contribuir para danos induzido pelo IAM nesses órgãos. Consistentemente, o PS mitigou o estresse oxidativo e restaurou as defesas antioxidantes (Glutationa - GSH nos pulmões: SHAM = 0,79 \pm 0,07; IAM = 0,67 \pm 0,05; IAM + PS $=0,86 \pm 0,14 ; p<0,05)$, indicando seu papel protetor neste cenário.

Conclusão: Nosso trabalho evidencia o potencial do uso de PS como abordagem terapêutica adjuvante após IAM para proteção dos tecidos pulmonares e cardíacos direitos.

Palavras-chave: Antioxidantes; Estresse Oxidativo; Óxido Nítrico Sintase; Homeostase; Hormese,; Pterostilbeno; NADPH Oxidases; Infarto do Miocárdio; Ratos.

\begin{abstract}
Background: Pterostilbene (PS), a natural and antioxidant polyphenolic compound emerges as a promising intervention in improving the myocardial infarction (MI) damages.

Objective: This study aimed to evaluate PS actions in promoting redox homeostasis in lungs and right ventricle (RV) of infarcted animals.

Methods: Male Wistar rats (60 day-old) were randomized into three groups: SHAM, MI (infarcted), and MI+PS (MI+pterostilbene). Seven days after MI procedure, rats were treated with PS $(100 \mathrm{mg} / \mathrm{kg} /$ day $)$ via gavage for eight days. Animals were euthanized and the lungs and RV were harvested for analyses of redox balance (Differences were considered significant when $p<0.05$ ).
\end{abstract}

Results: Our results show that MI triggers a redox disruption scenario in RV and lungs, which can contribute to MI-induced damage on these organs. Consistently, PS mitigated oxidative stress and restored antioxidant defenses (GSH in lungs: SHAM=0.79 $\pm 0.07 ; \mathrm{MI}=0.67 \pm 0.05$; $M I+P S=0.86 \pm 0.14 ; p<0.05)$, indicating its protective role in this scenario.

Conclusion: Our work evidences the PS potential use as an adjuvant therapeutic approach after MI focusing on protecting pulmonary and right-sided heart tissues.

Keywords: Antioxidants; Oxidative Stress, Nitric Oxidase Synthase; Homeostasis; Hormesis; Pterostilbene; NADPH Oxidases; Myocardial Infarction; Rats.

Full texts in English - https://abccardiol.org/en/

Correspondência: Alex Sander da Rosa Araújo •

Universidade Federal do Rio Grande do Sul - Sarmento Leite, 500. CEP 90040-060, Porto Alegre, RS - Brasil

E-mail: alex.rosa@ufrgs.br

Artigo recebido em 28/10/2020, revisado em 21/02/2021, aceito em 24/03/2021

DOI: https://doi.org/10.36660/abc.20201155 


\section{Introdução}

O infarto agudo do miocárdio (IAM), evento agudo que ocorre quando o fluxo sanguíneo coronariano é interrompido, culmina em alterações hemodinâmicas, neuro-humorais e metabólicas que podem impactar negativamente a função pulmonar. ${ }^{1,2}$ A remodelação cardíaca adversa pós-IAM induz modificação da geometria ventricular e deslocamento dos folhetos da válvula mitral, o que prejudica seu processo de fechamento e causa modificações prejudiciais que afetam ambos os ventrículos. Na verdade, o IAM ventricular esquerdo com regurgitação mitral pode levar a alterações hemodinâmicas nos vasos pulmonares, refletindo em última instância no aumento da pressão arterial pulmonar. Todos esses distúrbios podem desencadear hipertensão pulmonar secundária à cardiopatia esquerda. ${ }^{3}$ Nesse cenário, o aumento da resistência vascular pulmonar (RVP) compromete a câmara cardíaca direita em decorrência da pós-carga elevada do ventrículo direito (VD), levando ao aumento da espessura da parede e diminuição da contratilidade. Essas alterações culminam em resposta adaptative ruim, caracterizada por dilatação, disfunção e falência do ventrículo direito. ${ }^{4,5}$

Importantes mediadores associados ao dano cardiopulmonar induzido pelo infarto são as espécies reativas de oxigênio (ROS), cujas principais fontes são as NADPH oxidases, a xantina oxidase e as mitocôndrias. ${ }^{6-8}$ Nesse sentido, o sistema enzimático antioxidante - constituído principalmente por superóxido dismutase (SOD), catalase (CAT) e glutationa peroxidase (GPx) - é o mecanismo central de defesa contra o dano celular induzido por ROS. ${ }^{9}$ Além do sistema enzimático antioxidante, os tecidos também podem recrutar antioxidantes não enzimáticos, como a glutationa reduzida. ${ }^{10,11}$ No entanto, foi relatado que a resposta antioxidante do VD é reduzida após o IAM. ${ }^{12}$ Nessa situação, a resposta contrarregulatória contra a ruptura da homeostase redox pode ser coordenada pelo fator de transcrição antioxidante, conhecido como fator nuclear eritroide 2 relacionado ao fator 2 (Nrf2). ${ }^{13}$ De fato, o Nrf2 regula a expressão de várias proteínas redox por meio da indução de elementos de resposta antioxidante (ERA), principalmente em condições de estresse oxidativo, ${ }^{14}$ e pode ser ativado por antioxidantes naturais, como o pterostilbeno (PS). ${ }^{15}$

O PS é um composto encontrado em uma grande variedade de frutas vermelhas, como mirtilos (Vaccinium spp.) E uvas (Vitis spp.). Quimicamente, corresponde ao resveratrol dimetilado (trans-3,5-dimetoxi-4'-hidroxiestilbeno), diferenciando-se dele por sua maior lipofilicidade. ${ }^{15} \mathrm{O}$ mecanismo de ação dos estilbenos tem sido relacionado à redução dos níveis de ROS, como peróxido de hidrogênio e ânions superóxidos, bem como ao aumento da disponibilidade intracelular de antioxidantes enzimáticos e não enzimáticos. ${ }^{16}$ Nosso grupo relatou melhora nos parâmetros morfológicos do ventrículo esquerdo (VE) e nos marcadores de estresse oxidativo em ratos infartados tratados com PS (100 mg/kg/dia). ${ }^{15}$ No entanto, não existem estudos que avaliem se o composto pode causar uma atenuação dos danos induzidos pelo infarto nos pulmões e VD. Diante do exposto, o objetivo deste estudo foi avaliar o impacto do IAM sobre o estresse oxidativo no tecido pulmonar e no VD, e verificar se a administração de PS poderia melhorar a homeostase redox nesses órgãos.

\section{Métodos}

\section{Química}

O PS foi adquirido da Changsha Organic Herb (Changsha, China). A hidroxipropil--ciclodextrina (HPCD) foi fornecida pela Roquette Frères (Lestrem, França). A preparação e complexação do PS com HPCD para aumentar sua solubilidade em água foi conduzida como descrito previamente. ${ }^{15}$

\section{Ética}

Ratos Wistar machos (60 dias de idade) foram obtidos no Centro de Reprodução e Experimentação de Animais de Laboratório da Universidade Federal do Rio Grande do Sul. Os animais foram alocados em caixas de polipropileno (340 x $200 \times 410$ mm) com três/quatro animais por gaiola. Foram mantidos em condições padrão: temperatura de 20$25^{\circ} \mathrm{C}$, ciclos claro-escuro de 12 horas e umidade relativa de $70 \%$. Água e rações comerciais foram oferecidas ad libitum. O protocolo experimental foi realizado de acordo com as Diretrizes Internacionais para Uso e Cuidado de Animais de Laboratório e do Conselho Nacional de Controle de Experimentação Animal. O protocolo só foi iniciado após aprovação pelo Comitê de Ética para Experimentação Animal da Universidade (\#35451).

\section{Desenho experimental}

Primeiramente, os animais foram divididos aleatoriamente em gaiolas e todas as avaliações foram realizadas às cegas. ${ }^{15}$ A cirurgia de infarto do miocárdio e a administração de PS foram realizadas de acordo com estudos anteriores do nosso grupo. ${ }^{17}$ A taxa de mortalidade durante o procedimento cirúrgico de infarto foi de $10 \%$. Após a cirurgia, 17 ratos foram alocados nos seguintes grupos: SHAM $(n=6)$, grupo infartado (IAM) $(n=5)$ e grupo infartado tratado com PS (IAM + PS) $(n=6)$. A avaliação do tamanho da área de infarto do miocárdio foi realizada por meio de ecocardiografia (Philips HD7 XE Ultrasound System com transdutor L2-13 MHz), no dia 7 do protocolo experimental. Após essa avaliação, o PS foi administrado (100mg / kg / dia, por gavagem) para o grupo IAM + PS, e uma solução veículo (solução aquosa por gavagem) foi dada aos grupos SHAM e IAM por 8 dias. Após o período de tratamento, os animais foram sacrificados; os pulmões e os VDs foram coletados para análises morfométricas e bioquímicas.

\section{Avaliação ecocardiográfica}

As análises ecocardiográficas (14 dias após o infarto) foram realizadas usando o sistema EnVisor Philips (Andover, $M A, E U A)$ com um transdutor de alta frequência e alta resolução (12-3 $\mathrm{MHz}$ ) por um operador treinado e com experiência em ecocardiografia de ratos, sem conhecimento do tratamento. ${ }^{18}$ Os animais foram anestesiados (cetamina $90 \mathrm{mg} / \mathrm{kg}$; xilazina $20 \mathrm{mg} / \mathrm{kg}$, i.p.) e colocados em decúbito lateral para obtenção das imagens. As imagens do ventrículo esquerdo (VE) foram avaliadas em três planos: basal, médio e apical. Os valores de encurtamento fracionário (EF) do VE foram obtidos pela seguinte equação: 
LVFS $=$ DD - DS / DD $\times 100$ (diâmetro diastólico - DD diâmetro sistólico - DS). Os volumes sistólico e diastólico final do VE (VSF e VDF) foram medidos conforme descrito anteriormente. ${ }^{18} \mathrm{O}$ débito sistólico (SO) foi calculado como SO = VDF - VSF. ${ }^{19}$ Em cada plano transversal ecocardiográfico (basal, médio e apical), o arco correspondente aos segmentos com infarto (regiões ou segmentos do miocárdio apresentando uma das seguintes alterações na cinética miocárdica: movimento sistólico acinesia e/ou região de hipocinesia - RHC) foi medido quanto ao perímetro infartado. ${ }^{18,19} \mathrm{~A}$ avaliação do perímetro infartado foi então usada para estimar o tamanho do infarto do miocárdio (R.B. Teixeira et al. Life Sciences 196 (2018) 93-101).

\section{Análise morfométrica dos ventrículos esquerdo e direito} e pulmões

A eutanásia foi realizada por sobrecarga anestésica (cetamina $90 \mathrm{mg} / \mathrm{kg}$ e xilazina $10 \mathrm{mg} / \mathrm{kg}$, por via intraperitonial) e confirmada por luxação cervical. Após a eutanásia, os pulmões, o VE e o VD foram utilizados para medidas morfométricas e bioquímicas. O pulmão esquerdo foi utilizado para determinar a razão pulmão/peso corporal, a fim de avaliar a congestão pulmonar. Para a realização dos índices de hipertrofia dos ventrículos direito e esquerdo, foram calculadas as razões ventrículo/peso corporal e ventrículo/ comprimento da tíbia. ${ }^{20}$

\section{Preparação de homogenatos de pulmão e ventrículo direito}

O pulmão direito foi preparado para as seguintes análises de estresse oxidativo: ROS total, peroxidação lipídica, glutationa total, glutationa reduzida, concentração de tiol, atividades de enzimas antioxidantes e expressão da proteína Nrf2. O VD foi homogeneizado para avaliar as atividades de NADPH oxidase e óxido nítrico-sintase, níveis de sulfidrila e imunoconteúdo de xantina oxidase. A homogeneização do pulmão e do VD foi realizada por 40 segundos com Ultra-Turrax (OMNI Tissue Homogenizer, OMNI International, EUA) na presença de $\mathrm{KCl}$ $1,15 \%$ (5 mL/g tecido) e fluoreto de fenilmetilsulfonil 100 $\mathrm{mmol} / \mathrm{L}$ (PMSF). As amostras foram centrifugadas (20 minutos a $10.000 \times$ g a $^{\circ} \mathrm{C}$ ), e o sobrenadante foi coletado e armazenado a $80^{\circ} \mathrm{C}$ até a análise. ${ }^{21}$ As análises bioquímicas foram realizadas por um pesquisador que desconhecia o tratamento.

\section{Avaliação de estresse oxidativo}

No tecido pulmonar, a concentração total de ROS foi determinada pelo método de fluorescência por meio da reação com diacetato de dicloro fluoresceína (DCFH-DA) (Sigma-Aldrich, EUA). Os dados foram expressos em pmol/ mg de proteína. ${ }^{22}$ A peroxidação lipídica foi medida pela reação dos produtos da oxidação com as substâncias reativas ao ácido tiobarbitúrico (TBARS) e os resultados apresentados em nmol/mg de proteína. ${ }^{23}$ Os níveis de glutationa total (GSH total) e dissulfeto de glutationa (GSSG) foram determinados pela redução de 5,5-ditiobis (ácido 2-nitrobenzóico) (DTNB) pela nicotinamida adenina dinucleotídeo fosfato (NADPH) catalisada pela glutationa redutase. Os dados são expressos como $\mu \mathrm{mol} / \mathrm{min} / \mathrm{mg}$ de tecido. ${ }^{24}$

\section{Atividade NADPH Oxidase}

A atividade da enzima NADPH oxidase foi determinada pela avaliação do consumo de NADPH a $340 \mathrm{~nm}$. Os resultados foram expressos em nanomoles de $\mathrm{NADPH}$ por minuto por miligrama de proteína $(\mathrm{nmol} / \mathrm{min} / \mathrm{mg}$ de proteína). ${ }^{25}$

\section{Determinação de antioxidantes enzimáticos e não enzimáticos}

A atividade da SOD foi determinada por meio da inibição da auto-oxidação do pirogalol, e os resultados foram expressos em unidades de SOD/mg de proteína. ${ }^{26} \mathrm{~A}$ avaliação da atividade da CAT foi baseada no consumo de peróxido de hidrogênio, monitorando-se o decaimento da absorbância em $240 \mathrm{~nm}$. Os resultados foram expressos em $\mathrm{pmol} / \mathrm{min} /$ mg de proteína. ${ }^{27} \mathrm{~A}$ atividade da GPx foi estimada a partir da oxidação do NADPH, que foi acoplada à reação de reciclagem de GSSG para GSH, avaliada em $340 \mathrm{~nm}$. Os resultados foram expressos em nmol/mg de proteína. ${ }^{28}$ A quantidade total de grupos sulfidrila no tecido pulmonar foi determinada pela reação dos grupos tiol com DTNB. A concentração de grupos sulfidrila totais foi expressa em nmol TNB/mg proteína. ${ }^{29}$ A concentração de proteína foi medida pelo método de Lowry. ${ }^{30}$

\section{Atividade de óxido nítrico-sintase}

A atividade da enzima óxido nítrico-sintase foi avaliada medindo-se a conversão da oxihemoglobina ( $\mathrm{HbO} 2) \mathrm{em}$ metemoglobina, induzida pelo óxido nítrico, conforme descrito anteriormente. Os valores são expressos em nmol $\mathrm{NO} / \mathrm{min} / \mathrm{mg}$ de proteína. ${ }^{31}$

\section{Avaliação do conteúdo imunológico de Nrf2 e xantina oxidase}

O conteúdo imunológico de Nrf2 e xantina oxidase foram determinados por Western blot, conforme descrito anteriormente. ${ }^{32}$ Os anticorpos Nrf2 e a xantina oxidase foram usados como anticorpos primários (Santa Cruz Biotechnology, Santa Cruz, CA, EUA). Os anticorpos primários foram detectados por meio de anticorpos secundários conjugados com globulina de coelho anti-rato conjugada com peroxidase de rábano silvestre. As membranas foram desenvolvidas com reagentes de quimioluminescência. As autorradiografias foram escaneadas e as bandas foram medidas por meio de um software densitômetro (Imagemaster VDS Cl, Amersham Biosciences Europe, IT). As bandas de pesos moleculares de Nrf2 e xantina oxidase foram determinadas por referência a um marcador de peso molecular padrão (RPN 800 rainbow full range Bio-Rad, CA, EUA). Os resultados foram normalizados pelo método de Ponceau. ${ }^{33}$

\section{Análise estatística}

O cálculo do tamanho da amostra considerou uma probabilidade de erro $=0,05$ e testou o poder estatístico (probabilidade de erro 1-) =0,90. A distribuição dos dados foi avaliada pelo teste de Shapiro-Wilk. Como os dados apresentaram distribuição normal, os resultados foram analisados por ANOVA one-way com o teste post-hoc de Student-Newman-Keuls para detectar diferenças entre os 
grupos, e os resultados foram expressos em média \pm desvio padrão (DP). As diferenças foram consideradas significativas quando $p<0,05$. Os dados foram analisados usando o software Sigma Plot (Jandel, Scientific Co, v. 11.0, San Jose, CA, EUA).

\section{Resultados}

\section{Resultados morfométricos}

Os ratos infartados de ambos os grupos (IAM e IAM + PS) tiveram um perímetro de infarto semelhante. Isso indica que não há diferenças entre os grupos infartados em termos de tamanho da lesão. A congestão pulmonar não diferiu entre os grupos experimentais. Da mesma forma, não houve alteração dos índices de hipertrofia do ventrículo direito, calculados pelas razões ventrículo direito/peso corporal e ventrículo direito/comprimento da tíbia, bem como do peso do ventrículo direito entre os grupos (Tabela 1).

\section{Parâmetros ecocardiográficos}

Na avaliação dos parâmetros morfológicos do ventrículo esquerdo, tanto o grupo IAM quanto o IAM + PS apresentaram aumento dos volumes sistólico e diastólico final em comparação ao grupo SHAM, indicando dilatação ventricular. Porém, os animais IAM + PS apresentaram aumento do volume sistólico final mais discreto em relação ao IAM. O débito sistólico e a frequência cardíaca não foram diferentes entre os grupos. A fração de encurtamento do ventrículo esquerdo, que indica sua contratilidade, diminuiu nos grupos IAM e IAM + PS em relação aos animais SHAM, indicando piora na função sistólica desta câmara, e a administração de PS não foi eficaz para melhorar esse parâmetro. Em relação ao perímetro infartado, não houve diferença entre os grupos IAM e IAM + PS (Tabela 2).

Níveis de ROS, peroxidação lipídica e resposta antioxidante no tecido pulmonar

O estresse oxidativo foi medido por meio da produção de dicloro fluoresceína (DCF) (um indicador dos níveis totais de ROS) e TBARS (um indicador de peroxidação lipídica) no tecido pulmonar. Em relação aos ROS totais, o grupo IAM + PS apresentou níveis aumentados em comparação aos grupos SHAM e IAM $(p<0,05)$ (Figura 1A). No entanto, nenhuma diferença foi encontrada entre IAM e SHAM. Embora os níveis de espécies reativas tenham aumentado no grupo IAM + PS, a peroxidação lipídica diminuiu no tecido pulmonar desses animais em comparação com o grupo IAM $(\mathrm{P}<0,05)$. Além disso, a peroxidação lipídica no grupo IAM + PS não foi diferente em relação ao grupo SHAM, indicando redução do dano oxidativo promovida pela administração de PS (Figura 1B).

Tabela 1 - Resultados morfométricos do pulmão e dos ventrículos esquerdo e direito

\begin{tabular}{llll}
\hline & SHAM $(\mathbf{n}=5)$ & IAM $(\mathbf{n = 5})$ & IAM+PS $(\mathbf{n = 6})$ \\
\hline Pulmão/peso corporal $(\mathrm{g} / \mathrm{g})$ & $4,01 \pm 0,99$ & $5,99 \pm 0,44 a$ & $5,24 \pm 1,21$ \\
\hline VD $(\mathrm{g})$ & $0,17 \pm 0,037$ & $0,20 \pm 0,02$ & $0,24 \pm 0,07$ \\
\hline VD/comprimento da tíbia $(\mathrm{g} / \mathrm{cm})$ & $0,48 \pm 0,09$ & $0,57 \pm 0,07$ & $0,66 \pm 0,19$ \\
\hline VD/peso corporal $(\mathrm{mg} / \mathrm{g})$ & $0,50 \pm 0,11$ & $0,60 \pm 0,04$ & $0,70 \pm 0,23$ \\
\hline VE $(\mathrm{g})$ & $0,75 \pm 0,06$ & $0,75 \pm 0,08$ & $2,73 \pm 0,07$ \\
\hline VE/comprimento da tíbia $(\mathrm{g} / \mathrm{cm})$ & $2,05 \pm 0,19$ & $2,09 \pm 0,22$ & $2,00 \pm 0,18$ \\
\hline VE/peso corporal $(\mathrm{mg} / \mathrm{g})$ & $2,15 \pm 0,24$ & $2,21 \pm 0,22$ & $2,11 \pm 0,15$ \\
\hline
\end{tabular}

Dados apresentados como média \pm DP. ANOVA unilateral com o teste post-hoc de Student-Newman-Keuls. ap<0,05 vs SHAM. VD: ventrículo direito;

VE: ventrículo esquerdo; SHAM: grupo controle; IAM: infarto do miocárdio; IAM + PS: infarto do miocárdio + pterostilbeno.

Tabela 2 - Avaliação ecocardiográfica do ventrículo esquerdo

\begin{tabular}{|c|c|c|c|}
\hline & SHAM $(n=6)$ & IAM $(n=5)$ & $I A M+P S(n=6)$ \\
\hline VSFVE (mL) & $0,08 \pm 0,05$ & $0,46 \pm 0,15$ a & $0,32 \pm 0,06 a b$ \\
\hline VDFVE (mL) & $0,29 \pm 0,09$ & $0,68 \pm 0,14 \mathrm{a}$ & $0,57 \pm 0,06 \mathrm{a}$ \\
\hline Saída sistólica (mL) & $0,21 \pm 0,04$ & $0,22 \pm 0,03$ & $0,24 \pm 0,04$ \\
\hline Frequência cardíaca (bpm) & $249 \pm 16$ & $242 \pm 15$ & $237 \pm 27$ \\
\hline Fração de encurtamento (\%) & $51,5 \pm 5,4$ & $15,8 \pm 1,7 a$ & $17,0 \pm 2,9 a$ \\
\hline Perímetro infartado (cm) & ----------- & $1,81 \pm 0,45$ & $1,60 \pm 0,22$ \\
\hline
\end{tabular}

Dados apresentados como média \pm DP. ANOVA unilateral com o teste post-hoc de Student-Newman-Keuls. ap <0,05 vs SHAM; bp <0,05 vs IAM. VSFVE: Volume sistólico final do ventrículo esquerdo; VDFVE: Volume diastólico final do ventrículo esquerdo; SHAM: Grupo controle; IAM: grupo infarto do miocárdio; IAM + PS: infarto do miocárdio + pterostilbeno. o perímetro infartado é um parâmetro ecocardiográfico de medição do tamanho do infarto. 


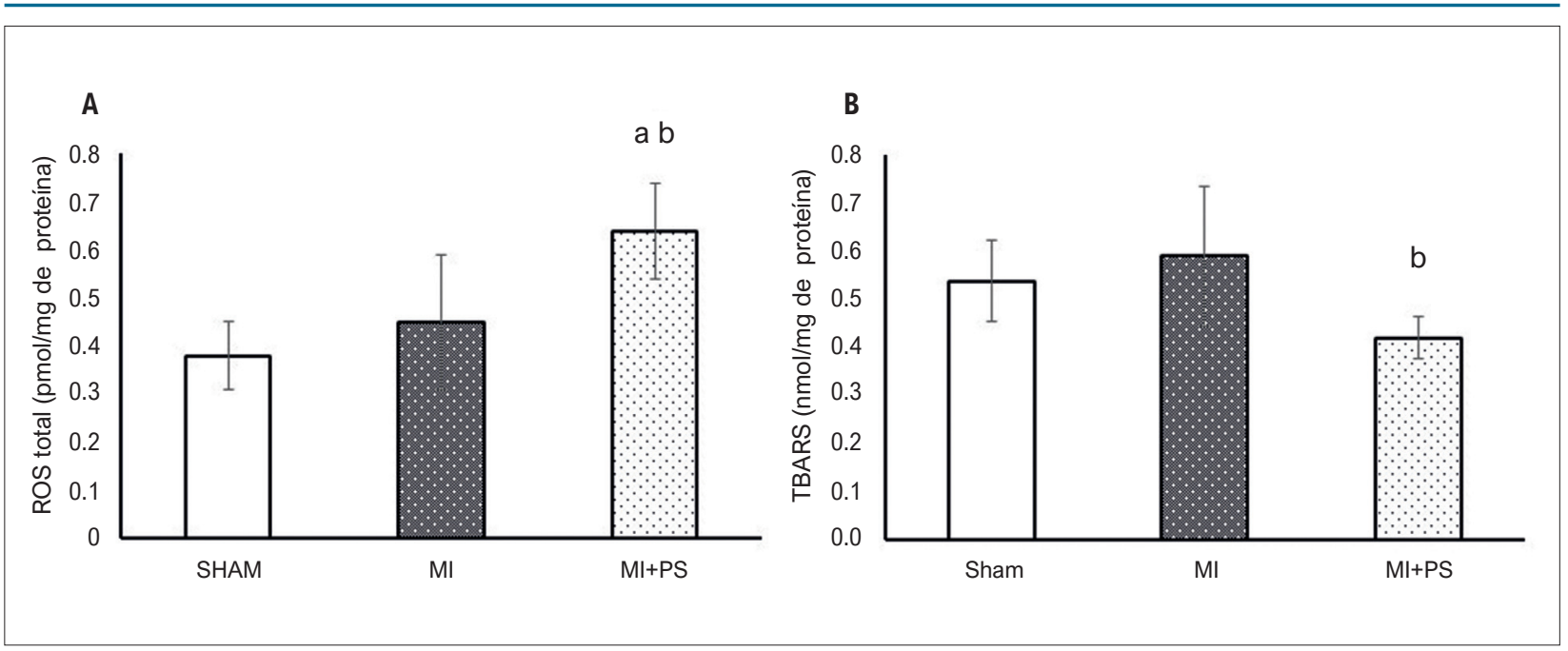

Figura 1 - Estresse oxidativo pulmonar A) Concentração total de espécies reativas de oxigênio; B) Substâncias reativas ao ácido tiobarbitúrico. Dados expressos como média \pm DP. ANOVA unilateral com o teste post-hoc de Student-Newman-Keuls. aP<0,05 vs SHAM; bP<0,05 vs IAM. SHAM: Grupo controle; IAM: grupo infarto do miocárdio; IAM+ PS: infarto do miocárdio + pterostilbeno.

Em termos de defesas antioxidantes não enzimáticas, nenhuma mudança significativa nos níveis de GSH em ratos com IAM foi observada em comparação com ratos SHAM. Porém, o tratamento com PS teve efeito positivo nos pulmões dos animais IAM + PS, uma vez que os níveis de GSH aumentaram neste grupo quando comparados com SHAM e IAM $(\mathrm{p}<0,05)$ (Tabela 3). No entanto, os níveis de GSSG, as relações GSH/Glutationa Total e GSSG/Glutationa Total não apresentaram diferenças entre os grupos.

Além da GSH, a administração de PS também parece melhorar as defesas antioxidantes enzimáticas nos pulmões. A atividade SOD foi reduzida no grupo IAM em comparação com o grupo SHAM. No entanto, essa atividade enzimática foi recuperada pelo PS ( $p<0,05)$ (Figura 2A). A CAT estava aumentada no grupo IAM + PS em relação aos grupos SHAM e IAM $(p<0,05)$ (Figura 2B). $\mathrm{A}$ atividade da GPx e o $\mathrm{SH}$ total, entretanto, não se alteraram entre os grupos (Figuras 2C e 2D, respectivamente).

\section{Expressão da proteína Nrf2 no tecido pulmonar}

A expressão de Nrf2 pode estar envolvida nos efeitos antioxidantes do PS. A Nrf2 é uma proteína relacionada com a regulação da transcrição de enzimas antioxidantes e pode ser estimulada por moléculas como os compostos fenólicos. De fato, nossos dados mostraram que o tratamento com PS promoveu um aumento significativo na expressão da proteína Nrf2 no grupo IAM + PS em comparação ao grupo IAM $(p<0,05)$. No entanto, não houve diferença na expressão de Nrf2 entre os grupos SHAM e IAM (Figura 3).

\section{Avaliação da Xantina Oxidase e NADPH Oxidase no VD}

O infarto do miocárdio estimulou enzimas pró-oxidantes, que foram atenuadas pela administração de PS. Em relação a isso, a expressão da proteína xantina oxidase foi aumentada no VD de animais com IAM em relação aos demais grupos $(p<0,05)$. No entanto, no grupo IAM + PS, os níveis de xantina oxidase não foram diferentes do grupo SHAM, o que indica uma atenuação dessa enzima pró-oxidante em animais infartados tratados com PS. Da mesma forma, houve um aumento na atividade da NADPH oxidase em animais infartados (IAM) em comparação com os do grupo SHAM, o que pareceu reduzido no grupo IAM + PS ( $p<0,05)$, comprovando a contribuição do tratamento com PS na

Tabela 3 - Parâmetros redox nos pulmões

\begin{tabular}{lccc}
\hline & SHAM $(\mathbf{n}=\mathbf{6})$ & IAM (n=5) & IAM+PS (n=6) \\
\hline GSH $(\mu \mathrm{mol} / \mathrm{min} / \mathrm{mg}$ de tecido) & $0,79 \pm 0,07$ & $0,67 \pm 0,05$ & $0,86 \pm 0,14 \mathrm{~b}$ \\
\hline GSSG $(\mu \mathrm{mol} / \mathrm{min} / \mathrm{mg}$ de tecido) & $0,24 \pm 0,09$ & $0,46 \pm 0,20$ & $0,36 \pm 0,14$ \\
\hline Glutationa total $(\mu \mathrm{mol} / \mathrm{min} / \mathrm{mg}$ de tecido) & $1,27 \pm 0,13$ & $1,40 \pm 0,22$ & $0,49 \pm 0,12$ \\
\hline GSH/ Glutationa total & $0,63 \pm 0,11$ & $0,57 \pm 0,15$ & $0,19 \pm 0,08$ \\
\hline GSSG/ Glutationa total & $0,18 \pm 0,05$ & $0,29 \pm 0,17$ \\
\hline
\end{tabular}

Os dados são apresentados como média \pm DP. ANOVA unilateral com o teste post-hoc de Student-Newman-Keuls bp<0,05 vs IAM. SAHM: Grupo controle; IAM: grupo infarto do miocárdio; IAM + OS: infarto do miocárdio + pterostilbeno; GSH: glutationa reduzida; GSSG: glutationa oxidada (unidade em $\mu \mathrm{mol} / \mathrm{min} / \mathrm{mg}$ de tecido). 


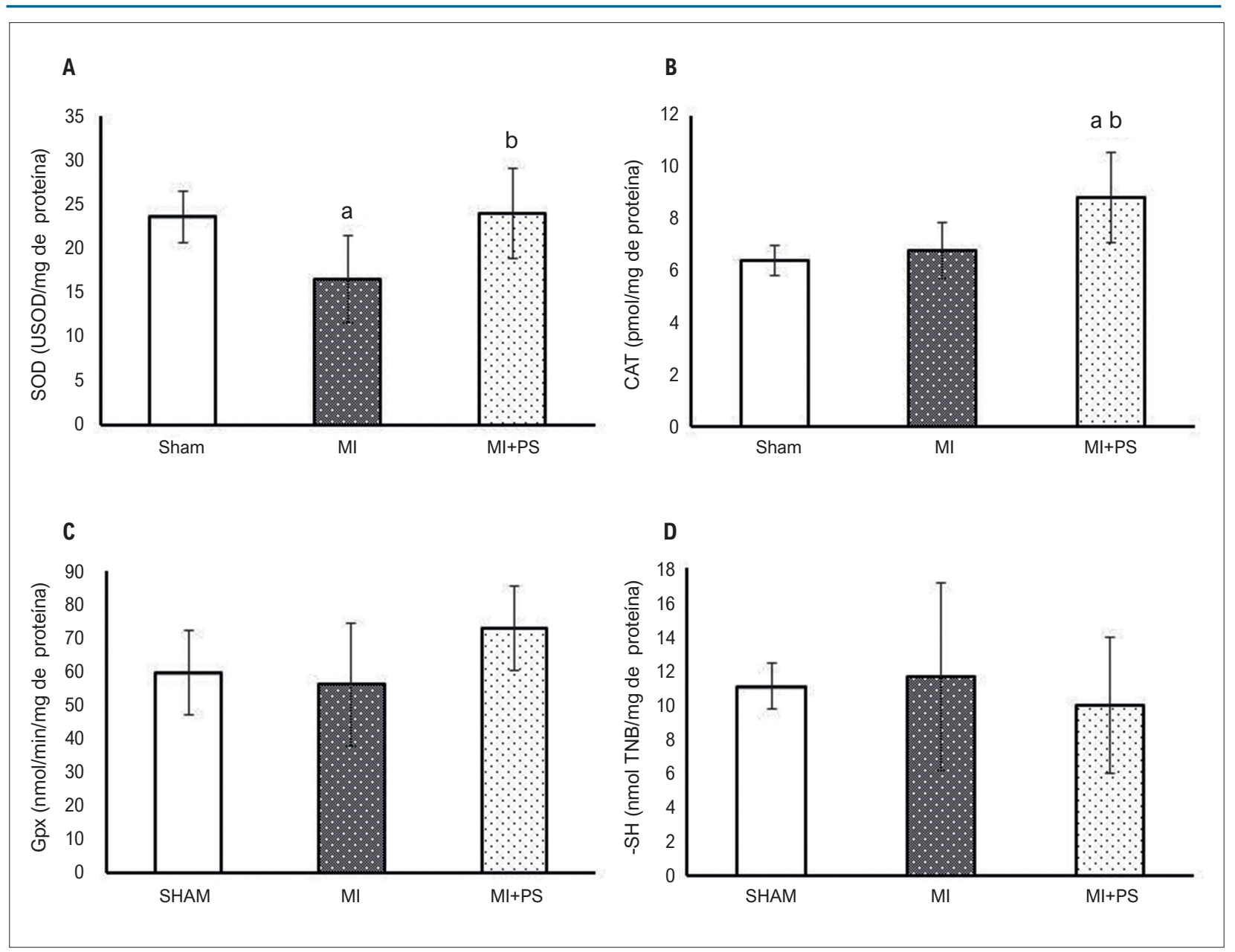

Figura 2 - Medições de antioxidantes pulmonares. A) Atividade da superóxido dismutase; B) Atividade da catalase; C) Atividade da glutationa peroxidase; D) Grupos sulfidrila totais. Dados expressos como média \pm DP. ANOVA unilateral com o teste post-hoc de Student-Newman-Keuls. aP<0,05 vs SHAM; bP<0,05 vs IAM. SHAM: Grupo controle; IAM: grupo infarto do miocárdio; IAM + PS: infarto do miocárdio + pterostilbeno.

redução da produção de radical ânion superóxido no VD (Figura 4A e 4B).

\section{Concentração de sulfidrila e atividade de NOS no VD}

A concentração de sulfidrila (grupos tiol relevantes como antioxidantes não enzimáticos) foi diminuída no grupo IAM em comparação com o SHAM $(p<0,05)$; entretanto, o grupo IAM + PS restabeleceu os níveis de sulfidrila $(p<0,05)$. Além disso, ratos infartados não tratados apresentaram redução da atividade da NOS quando comparados aos animais SHAM, enquanto o tratamento com PS induziu a recuperação dessa atividade enzimática ( $p<0,05)$ (Figura 5A e 5B).

\section{Discussão}

O principal achado deste estudo foi que o tratamento de ratos infartados com PS promoveu efeitos benéficos nos pulmões e no VD. Ao avaliar a morfologia e função do VE após o infarto, ambos os grupos infartados apresentaram dilatação cardíaca, refletida pelo aumento dos volumes cardíacos, e comprometimento da contratilidade, evidenciado pela diminuição da fração de encurtamento. A administração do PS, entretanto, atenuou o aumento do volume sistólico final, o que parece ser um resultado positivo, uma vez que o aumento do volume sistólico final pode estar relacionado ao desenvolvimento de congestão pulmonar. Na verdade, o tratamento com PS evitou a congestão pulmonar, conforme mostrado pela relação pulmão/peso corporal. O perímetro infartado não foi diferente entre os ratos IAM e IAM + PS, havendo homogeneidade de lesão cardíaca entre esses grupos. Em relação aos parâmetros morfométricos, nem o ventrículo direito nem o esquerdo dos grupos infartados apresentou hipertrofia. O fato de os animais terem sido avaliados apenas 14 dias após o infarto poderia explicar isso. Um estudo anterior ao nosso também não encontrou diferença nesses parâmetros. ${ }^{32}$

Os pulmões são os órgãos mais afetados pela insuficiência cardíaca, e a disfunção pulmonar é um fator-chave para desfechos clínicos ruins em pacientes infartados. ${ }^{34} \mathrm{No}$ entanto, nosso estudo não encontrou alterações nos níveis totais de ROS nos pulmões de ratos infartados. Por outro 


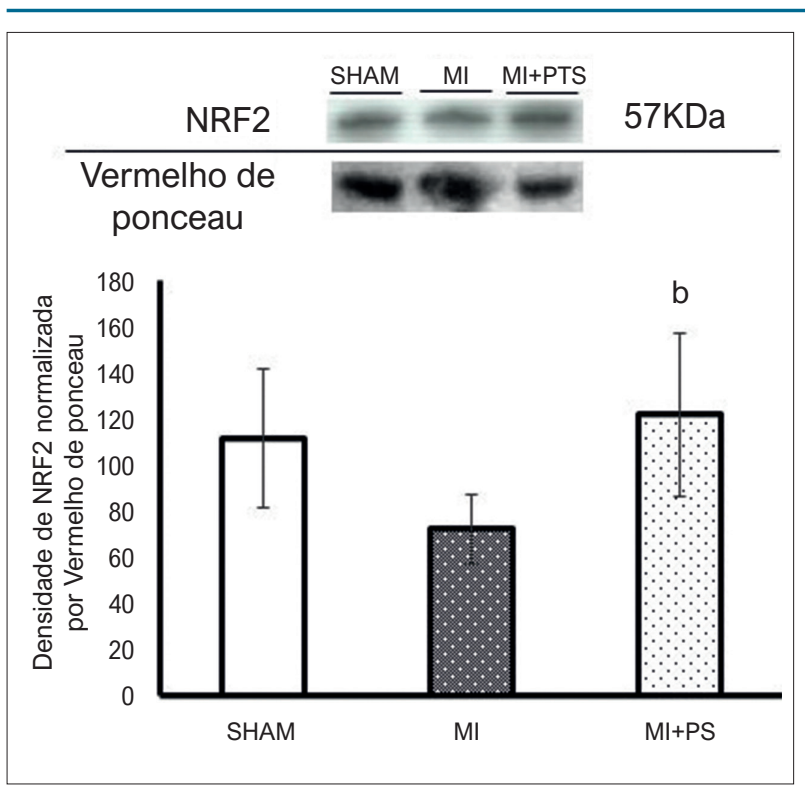

Figura 3 - Análise Western Blot da expressão de Nrf2 no pulmão. Gráfico representativo mostrando 1 banda para cada grupo experimental. Dados expressos como média \pm DP. ANOVA unilateral com o teste post-hoc de Student-Newman-Keuls. $b P<0,05$ vs IAM. SHAM: Grupo controle; IAM: grupo infarto do miocárdio; IAM + PS: infarto do miocárdio + pterostilbeno.

lado, os pulmões de animais IAM + PS apresentaram níveis aumentados de ROS, o que está de acordo com o papel relatado dos estilbenos na indução da produção de ROS in vitro. ${ }^{16} \mathrm{O}$ grupo IAM mostrou um aumento na peroxidação lipídica, indicando dano oxidativo nos pulmões. O grupo IAM + PS, entretanto, apresentou redução da lesão pulmonar induzida pela peroxidação lipídica, uma vez que os níveis de TBARS diminuíram. Uma possível hipótese é que esse aumento dos níveis de ROS ocasionado pela administração de PS poderia representar um mecanismo hormonal ${ }^{35}$ que leva ao aumento das defesas antioxidantes, evitando a peroxidação lipídica. De fato, estudos anteriores com outros compostos naturais que também apresentam efeito pró-oxidante, como o sulforafano, já reportaram esse mecanismo de proteção pela estimulação do sistema antioxidante. ${ }^{36} \mathrm{O}$ tratamento com PS pode induzir uma adaptação contra o aumento dos níveis de ROS por meio de alterações oxidativas celulares na atividade de SOD e CAT, duas enzimas importantes que pertencem à primeira linha de defesa contra o estresse oxidativo ${ }^{9} \mathrm{Em}$ nosso estudo, a redução da atividade da SOD nos pulmões do grupo IAM sugere proteção deficiente contra os radicais superóxido ânion, o que poderia causar um aumento do estresse oxidativo em estágios posteriores do IAM. ${ }^{17}$ Por outro lado, o tratamento com PS recuperou a atividade SOD no grupo IAM + PS, demonstrando seu efeito protetor na homeostase redox. Nossos resultados mostraram aumento da atividade CAT no tecido pulmonar de animais IAM + PS. Como o peróxido de hidrogênio pode reagir com metais como o ferro e produzir radicais hidroxila, ${ }^{37}$ esse aumento da atividade CAT no grupo IAM + PS surge como uma importante defesa contra a produção desse radical nos pulmões. Em termos de defesas não enzimáticas, foi encontrado um aumento na concentração de GSH nos pulmões de animais IAM + PS. GSH é o peptídeo antioxidante de baixo peso molecular mais prevalente ${ }^{38}$ e participa da regulação redox e da homeostase. ${ }^{39}$ Neste estudo, o aumento da concentração de GSH pode ter contribuído para a redução dos níveis de TBARS no grupo

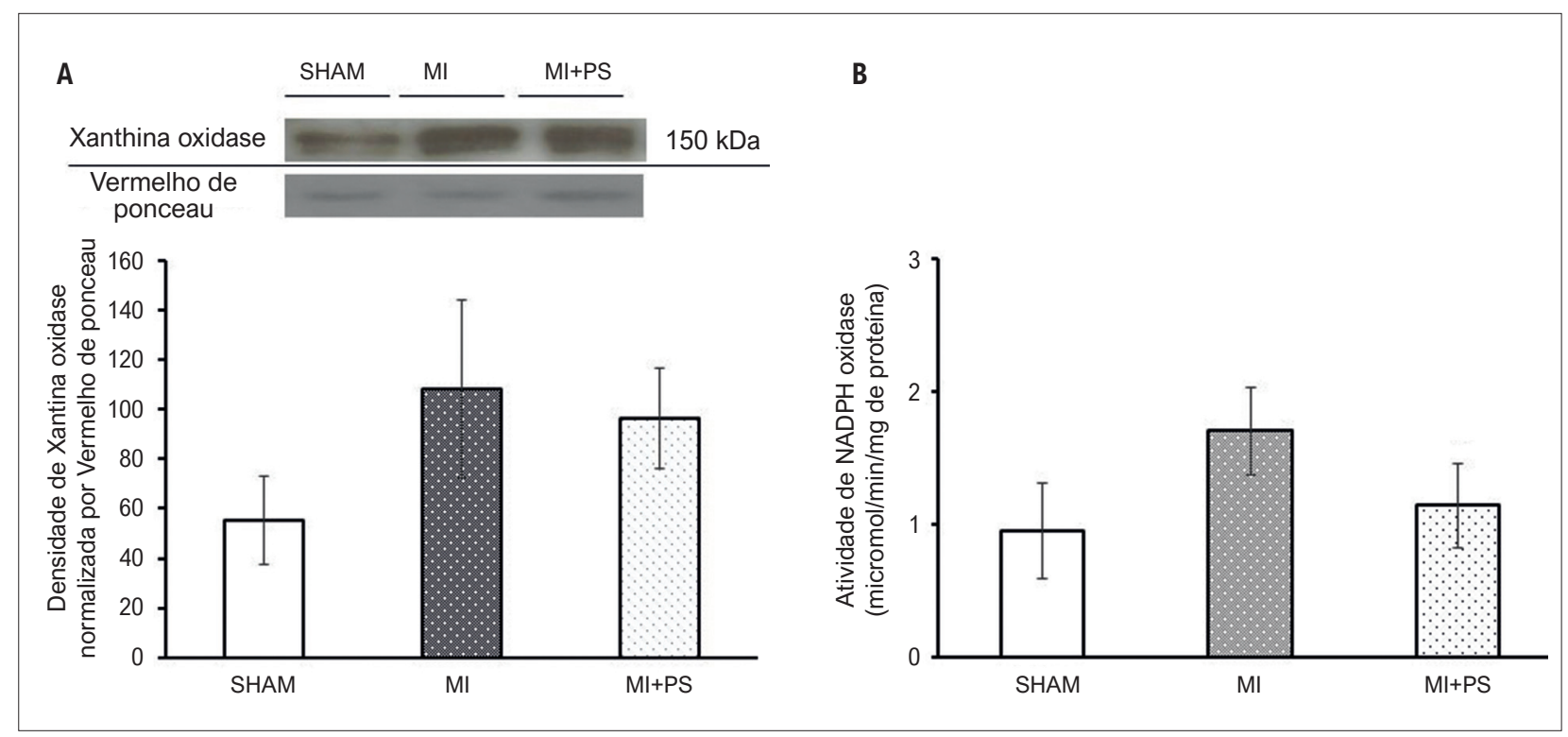

Figura 4 - Estresse oxidativo do ventrículo direito. A) Análise Western Blot da expressão da xantina oxidase. Gráfico representativo mostrando 1 banda para cada grupo experimental; B) Atividade de NADPH oxidases. Dados expressos como média \pm DP. ANOVA unilateral com o teste post-hoc de Student-Newman-Keuls. aP<0,05 vs SHAM; bP<0,05 vs IAM. SHAM: Grupo controle; IAM: grupo infarto do miocárdio; IAM + PS: infarto do miocárdio + pterostilbeno. 


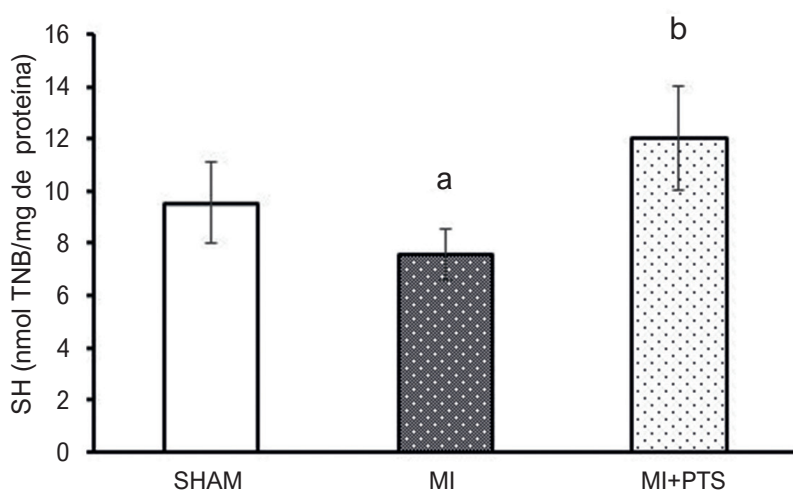

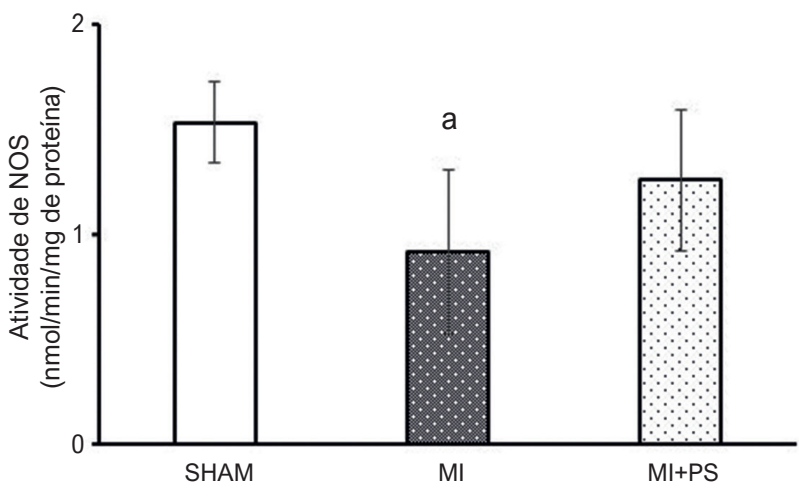

Figura $5-$ A) Grupos sulfidrila total do ventrículo direito; B) Atividade da óxido nítrico-sintase do ventrículo direito. Dados expressos como média \pm DP. ANOVA unilateral com o teste post-hoc de Student-Newman-Keuls. aP<0,05 vs SHAM; bP<0,05 vs IAM. SHAM: Grupo controle; IAM: grupo infarto do miocárdio; IAM + PS: infarto do miocárdio + pterostilbeno.

IAM + PS, diminuindo o estresse oxidativo nesses animais. Além da estimulação das defesas enzimáticas e não enzimáticas, o PS também foi capaz de induzir melhorias no perfil antioxidante dos tecidos pulmonares por meio da estimulação de proteínas citoprotetoras, como a Nrf2. ${ }^{40}$

Nesse contexto, a Nrf2 atua como um fator de transcrição sensível à redox, desempenhando um papel fundamental na resposta antioxidante pulmonar.40 Em situações de equilíbrio redox, a Nrf2 está ancorado na proteína 1, associada à ECH semelhante a Kelch (Keap1). Porém, quando há uma ruptura da homeostase redox, o complexo Nrf2-Keap1 se dissocia e libera Nrf2, que pode se translocar para o núcleo e iniciar a transcrição de moléculas antioxidantes. ${ }^{41}$ De fato, estudos têm demonstrado que o Nrf2 desempenha um papel importante na síntese de enzimas antioxidantes endógenas. Além disso, segundo Lacerda et al. ${ }^{15}$ o PS regula positivamente a expressão de Nrf2, o que aumenta o GSH celular e mitiga o dano oxidativo. ${ }^{15} \mathrm{Em}$ nosso estudo, os níveis de imunoconteúdo Nrf2 estavam aumentados nos pulmões de ratos IAM + PS, sugerindo que o PS induz a ativação de Nrf2, o que poderia explicar a melhora nas defesas antioxidantes e redução da peroxidação lipídica. Portanto, o PS mostrou ser protetor para os pulmões após o infarto, pois evitou a congestão pulmonar, aumentou as atividades de SOD e CAT, aumentou os níveis de GSH e evitou a peroxidação lipídica, além de induzir Nrf2, uma proteína citoprotetora.

O cenário pró-oxidativo do infarto do miocárdio afeta significativamente o VD. ${ }^{20}$ Nossos resultados mostraram que o infarto do miocárdio leva à expressão elevada da xantina oxidase nele. Wang et al. ${ }^{42}$ mostraram aumento dos níveis de xantina oxidase no coração 12 semanas após o infarto, associado à peroxidação lipídica e disfunção cardíaca. ${ }^{42}$ Além disso, a atividade da NADPH oxidase, importante fonte de ROS, também foi avaliada em nosso estudo. A atividade da enzima foi elevada em ratos IAM, e a administração de PS foi capaz de prevenir esse aumento. Os níveis elevados de xantina oxidase e a atividade da NADPH oxidase predispõem o VD ao aumento da concentração do ânion superóxido e, consequentemente, à depleção das reservas antioxidantes. Corroborando esses resultados, também encontramos níveis reduzidos de sulfidrila no grupo IAM. O grupo IAM + PS, entretanto, apresentou conteúdo aumentado de grupos tiol. No grupo IAM, a concentração elevada de ânion superóxido produzida por NADPH e xantina oxidase pode interferir desfavoravelmente no equilíbrio de ROS/NO no VD. Na verdade, nossos resultados mostraram atividade reduzida de NOS na câmara direita de animais IAM. A NOS é uma enzima relevante na produção de NO, que desempenha papel fundamental na cardioproteção. ${ }^{43}$ No presente estudo, a administração de PS evitou a redução da atividade de NOS que ocorreu no grupo IAM. No entanto, considerando o efeito desse composto no embotamento de enzimas pró-oxidantes, como a NADPH oxidase e a xantina oxidase, concomitante à recuperação parcial da atividade da NOS, o PS parece contribuir para a manutenção de um equilíbrio cardioprotetor ROS/NO no VD.

\section{Conclusões}

Em conclusão, a administração de PS promoveu efeitos benéficos nos pulmões de animais infartados, diminuindo a peroxidação lipídica e aumentando as defesas antioxidantes, como SOD, atividade de CAT e níveis de GSH. Também evitou o aumento da atividade da NADPH oxidase e da expressão da xantina oxidase no VD de animais infartados. Esses resultados provavelmente estão relacionados a uma melhora no equilíbrio ROS/NO nesta câmara. Portanto, nossos achados sugerem que o PS efetivamente tem efeitos protetores nos pulmões e no VD após IAM. 


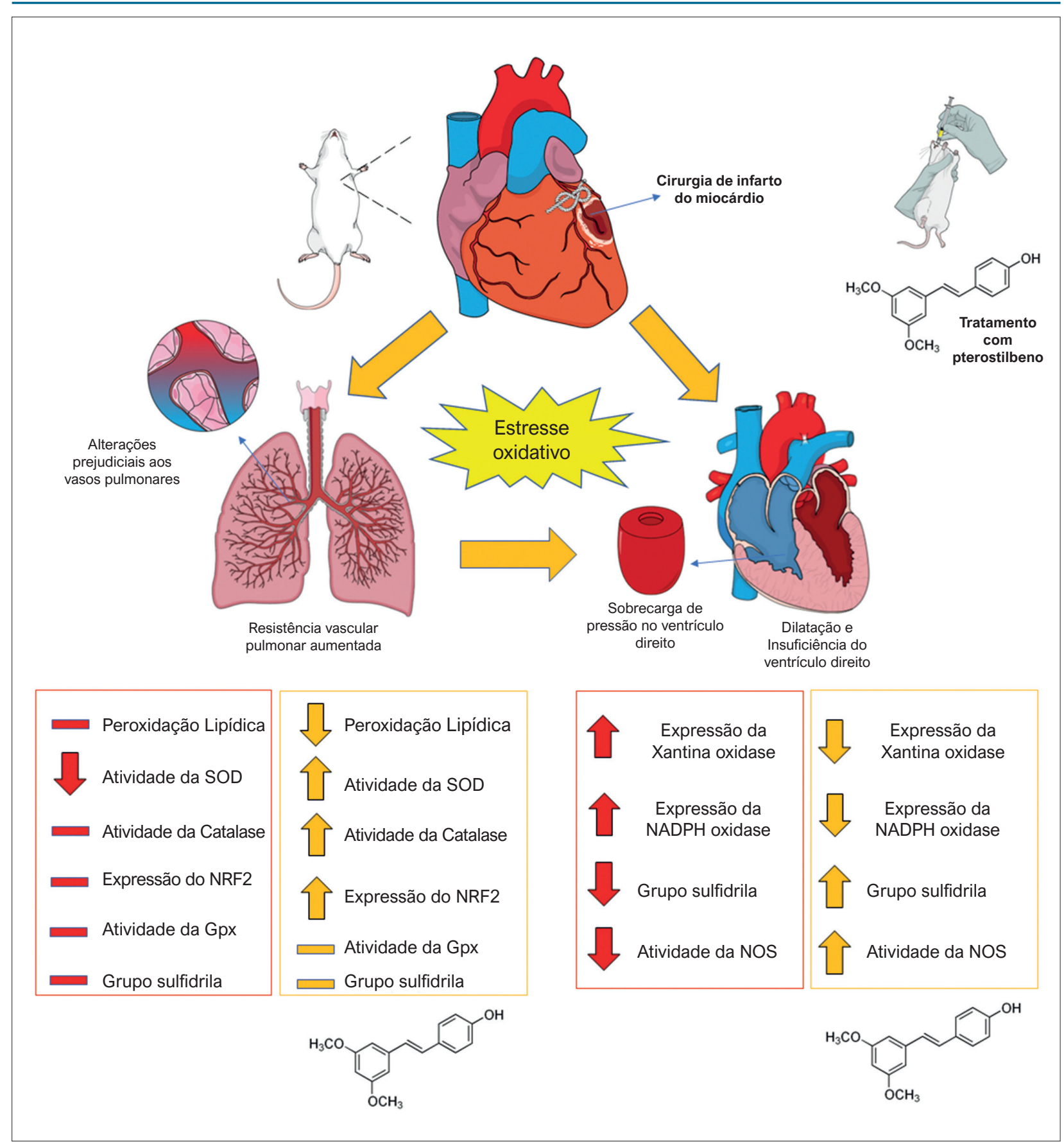

Figura 6 - Resumo gráfico

\section{Agradecimentos}

Este trabalho foi financiado pelo Conselho Nacional de Desenvolvimento Científico (CNPq) e pela Coordenação de Aperfeiçoamento de Pessoal de Nível Superior (CAPES).

\section{Contribuição dos autores}

Concepção e desenho da pesquisa: Tasca S, Lacerda D, Castro AL, Araújo ASR; Obtenção de dados: Tasca S, Campos
C, Bianchi SE; Análise e interpretação dos dados: Tasca S, Campos C, Lacerda D, Turck P; Análise estatística: Tasca S, Ortiz VD, Turck P; Obtenção de financiamento: Belló-Klein A, Bassani V, Araújo ASR; Redação do manuscrito: Tasca S; Revisão crítica do manuscrito quanto ao conteúdo intelectual importante: Castro AL, Belló-Klein A, Bassani V, Araújo ASR.

\section{Potencial conflito de interesse}

Não há conflito com o presente artigo 


\section{Fontes de financiamento}

O presente estudo não teve fontes de financiamento externas.

\section{Referências}

1. Bax JJ, Di Carli M, Narula J, Delgado V. Multimodality imaging in ischaemic heart failure. Lancet. 2019;393(10175):1056-70.

2. Chien CW, Wang CH, Chao ZH, Huang SK, Chen PE, Tung TH. Different treatments for acute myocardial infarction patients via outpatient clinics and emergency department. Medicine. 2019;98(2):e13883.

3. Jankowich M, Choudhary G. Endothelin-1 levels and cardiovascular events. Trends Cardiovasc Med 2019;30(1):1-8.

4. Greyson CR, Schwartz GG, Lu L, Ye S, Helmke S, Xu Y. Calpain inhibition attenuates right ventricular contractile dysfunction after acute pressure overload. J Mol Cell Cardiol. 2008;44(1):59-68.

5. Mann DL, Bristow MR. Mechanisms and models in heart failure: The biomechanical model and beyond. Circulation. 2005;111(21):2837-49.

6. Aydin S, Ugur K, Aydin S, Sahin I, Yardim M. Biomarkers in acute myocardia infarction: Current perspectives. Vasc Health RiskManag.;31(12) 2019;15:1-10

7. Zemskov E, Lu Q, Wojciech Ornatowski W, Klinger CN, Desai AA, Maltepe E, et al. Biomechanical forces and oxidative stress: Implications for pulmonary vascular disease. Antioxidants \& redox signaling. 2019;31(12):doi:10.1089/ ars.2018.1720.

8. Wang X, Shults NV, Suzuki YJ. Oxidative profiling of the failing right heart in rats with pulmonary hypertension. PloS one. 2017;12(5):e0176887.

9. Halliwell B, Gutteridge JM. The definition and measurement of antioxidants in biological systems. Free Radic Biol Med. 1995;18:125-6. doi:10.1016/0891-5849(95)-94457-3.

10. Comhair SA, Erzurum SC. Antioxidant responses to oxidant-mediated lung diseases. Am J Physiol Lung Cell Mol Physiol. 2002;283(2):L246-255.

11. Roy J, Galano JM, Durand T, Le Guennec JY, Lee JC. Physiological role of reactive oxygen species as promoters of natural defenses. FASEB journal : official publication of the Federation of American Societies for Experimental Biology. 2017;31(9):3729-45.

12. Hill MF, Singal PK. Right and left myocardial antioxidant responses during heart failure subsequent to myocardial infarction. Circulation. 1997;96(7):2414-20.

13. Shaw P, Chattopadhyay A. Nrf2-are signaling in cellular protection: Mechanism of action and the regulatory mechanisms. J Cell Physiol. 2020;235(4):3119-30.

14. Walters DM, Cho HY, Kleeberger SR. Oxidative stress and antioxidants in the pathogenesis of pulmonary fibrosis: A potential role for nrf2. Antioxidants \& redox signaling. 2008;10(2):321-32.

15. Lacerda D, Ortiz V, Turck P, Campos-Carraro C, Zimmer A, Teixeira R, et al. Stilbenoid pterostilbene complexed with cyclodextrin preserves left ventricular function after myocardial infarction in rats: Possible involvement of thiol proteins and modulation of phosphorylated gsk-3beta. Free Radic Res. 2018;52(9):988-99.

16. Treml J, Lelakova V, Smejkal K, Paulickova T, Labuda S, Granica S, et al. Antioxidant activity of selected stilbenoid derivatives in a cellular model system. Biomolecules. 2019;9:468.

17. Schenkel PC, Tavares AM, Fernandes RO, Diniz GP, Ludke AR, Ribeiro MF, et al. Time course of hydrogen peroxide-thioredoxin balance and its influence on the intracellular signalling in myocardial infarction. Exp Physiol. 2012;97(6):741-9.

18. de Castro AL, Tavares AV, Campos C, Fernandes RO, Siqueira R, Conzatti $A$, et al. Cardioprotective effects of thyroid hormones in a rat model of

\section{Vinculação acadêmica}

Este artigo é parte de dissertação de mestrado de Alex Sander da Rosa Araujo pela Universidade Federal do Rio Grande do Sul.

myocardial infarction are associated with oxidative stress reduction. Mo Cell Endocrnol.2014;391(1-2):22-9.

19. Nozawa E, Kanashiro RM, Murad N, Carvalho AC, Cravo SL, Campos O, et al. Performance of two-dimensional doppler echocardiography for the assessment of infarct size and left ventricular function in rats. Braz J MedBiol Res. 2006;39(5):687-95.

20. Corssac GB, de Castro AL, Tavares AV, Campos C, Fernandes RO, Ortiz VD et al. Thyroid hormones effects on oxidative stress and cardiac remodeling in the right ventricle of infarcted rats. Life Sci. 2016;146:109-16.

21. Llesuy SF, Milei J, Molina H, Boveris A, Milei S. Comparison of lipid peroxidation and myocardial damage induced by adriamycin and 4'-epiadriamycin in mice. Tumori. 1985;71(3):241-9.

22. LeBel CP, Ischiropoulos H, Bondy SC. Evaluation of the probe $2^{\prime}, 7^{\prime}$-dichlorofluorescin as an indicator of reactive oxygen species formation and oxidative stress. Chem Res Toxicol. 1992;5(2): 227-31.

23. Esterbauer $\mathrm{H}$, Cheeseman $\mathrm{KH}$. Determination of aldehydic lipid peroxidation products: Malonaldehyde and 4-hydroxynonenal. Meth Enzymol .1990;186(2): enzymology. 1990;186(2):407-21

24. Akerboom TP, Sies H. Assay of glutathione, glutathione disulfide, and glutathione mixed disulfides in biological samples. Meth Enzymol. $1981 ; 77: 373-82$

25. Wei Y, Sowers JR, Nistala R, Gong H, Uptergrove GM, Clark SE, et al. Angiotensin ii-induced nadph oxidase activation impairs insulin signaling in skeletal muscle cells. J Biol Chem.2006;281(46):35137-46.

26. Marklund SL. Superoxide dismutase isoenzymes in tissues and plasma from new zealand black mice, nude mice and normal balb/c mice. Mutation Res. $1985 ; 148(1-2): 129-34$

27. Aebi H. Catalase in vitro. Meth Enzymol. 1984;105:121-6.

28. Flohe L, Gunzler WA. Assays of glutathione peroxidase. Meth Enzymol. 1984:105:114-21.

29. Sedlak J, Lindsay RH. Estimation of total, protein-bound, and nonprotein sulfhydryl groups in tissue with ellman's reagent. Anal Biochem. 1968;25(1):192-205

30. Lowry OH, Rosebrough NJ, Farr AL, Randall RJ. Protein measurement with the folin phenol reagent. J Biol Chem; 1951;193(1):265-75.

31. Valdez LB, Zaobornyj T, Boveris A. Functional activity of mitochondrial nitric oxide synthase. Meth Enzymol. 2005;396:444-55.

32. de Castro AL, Fernandes RO, Ortiz VD, Campos C, Bonetto JH, Fernande TR, et al. Thyroid hormones improve cardiac function and decrease expression of pro-apoptotic proteins in the heart of rats 14 days after infarction. Apoptosis. 2016;21(2):184-94

33. Klein D, Kern RM, Sokol RZ. A method for quantification and correction of proteins after transfer to immobilization membranes. Biochem Mol Biol Int. 1995;36:(1)59-66

34. Yuan G, Han A, Wu J, Lu Y, Zhang D, Sun Y, et al. Bao yuan decoction and tao hong si wu decoction improve lung structural remodeling in a rat model of myocardial infarction: Possible involvement of suppression of inflammation and fibrosis and regulation of the tgf-beta1/smad 3 and nf-kappab pathways. BioscTrends. 2018;12(5):491-501

35. Mattson MP. Hormesis defined. Ageing Res Rev. 2008;7(1): 1-7. 
36. Fernandes RO, De Castro AL, Bonetto JH, Ortiz VD, Muller DD, CamposCarraro C, et al. Sulforaphane effects on postinfarction cardiac remodeling in rats: Modulation of redox-sensitive prosurvival and proapoptotic proteins. J Nutr Byochem. 2016;34:106-17.

37. Lee JC, Son YO, Choi KC, Jang YS. Hydrogen peroxide induces apoptosis of bjab cells due to formation of hydroxyl radicals via intracellular ironmediated fenton chemistry in glucose oxidase-mediated oxidative stress. Mol Cells. 2006;22(1):21-9.

38. Dincer Y, Alademir Z, Ilkova H, Akcay T. Susceptibility of glutatione and glutathione-related antioxidant activity to hydrogen peroxide in patients with type 2 diabetes: Effect of glycemic control. Clin Biochem.. 2002;35(4):297-301.

39. Mieyal JJ, Gallogly MM, Qanungo S, Sabens EA, Shelton MD. Molecular mechanisms and clinical implications of reversible protein s-glutathionylation. Antioxidants \& redox signaling. 2008;10(11):1941-88.

40. Trachootham D, Lu W, Ogasawara MA, Nilsa RD, Huang P. Redox regulation of cell survival. Antioxidants \& redox signaling. 2008;10(8):1343-74.

41. Campos C, de Castro AL, Tavares AM, Fernandes RO, Ortiz VD, Barboza TE, et al. Effect of free and nanoencapsulated copaiba oil on monocrotaline-induced pulmonary arterial hypertension. J Cardiovasc Pharmacol. 2017;69(2):79-85

42. Wang Z, Ding J, Luo X, Zhang S, Yang G, Zhu Q, et al. Effect of allopurinol on myocardial energy metabolism in chronic heart failure rats after myocardial infarct. Int Heart J. 2016;57(6):753-9.

43. de Castro AL, Tavares AV, Fernandes RO, Campos C, Conzatti A, Siqueira $\mathrm{R}$, et al. T3 and t4 decrease ros levels and increase endothelial nitric oxide synthase expression in the myocardium of infarcted rats. Mol Cell Biochem. 2015;408(1-2):235-43. 